\title{
An Algorithm That Identifies Coronary and Heart Failure Events In The Electronic Health Record
}

\author{
Jawali Jaranilla*1, Thomas E. Kottke ${ }^{1}$ and Courtney J. Baechler ${ }^{2}$ \\ ${ }^{1}$ Health Partners Institute for Education and Research, Minneapolis, MN, USA; ${ }^{2}$ University of Minnesota, Minneapolis, MN, USA
}

\section{Objective}

The objective of this project was to identify criteria that accurately categorize acute coronary and heart failure events exclusively with electronic health record data so that the medical record can be used for surveillance without manual record review.

\section{Introduction}

Surveillance to track the incidence, prevalence and treatment of disease is a fundamental task of public health. The advent of universal health care coverage in the United States and electronic health records could make the medical record a valuable disease surveillance tool. This can only happen, however, if the necessary data can be extracted from the medical record without manual review.

\section{Methods}

We serially compared 3 different computer algorithms to manual record review. The first two algorithms relied on ICD9CM codes, troponin levels, ECG data and echocardiographic data. The 3rd algorithm relied on a very detailed coding system, IMO statements, troponin levels and echocardiographic data.

\section{Results}

Cohen's Kappa for the initial algorithm was 0.47 (95\%CI 0.41 $0.54)$. Cohen's Kappa was $0.61(95 \%$ CI $0.55-0.68)$ for the second algorithm. Cohen's Kappa for the third algorithm was $0.99(95 \% \mathrm{CI}$ 0.98-1.00).

\section{Conclusions}

We conclude that electronic medical record data are sufficient to categorize coronary heart disease and heart failure events without manual record review. However, only moderate agreement with medical record review can be achieved when the classification is based on 4-digit ICD9CM codes because ICD9CM 410.9 includes myocardial infarction with ST elevation (STEMI) and myocardial infarction without ST elevation (nSTEMI). Nearly perfect agreement can be achieved using IMO statements, a more detailed coding system that tracks to ICD9, ICD10 and SnoMED-CT. IMO statements are available in many electronic medical record systems.

\section{Keywords}

validity; surveillance; coronary artery disease; heart failure; electronic medical record

\section{Acknowledgments}

Funding provided by the following: The HealthPartners Research Foundation (a partnership grant to TEK); The Heart Disease and Stroke Prevention Unit at the Minnesota Department of Health from a Capacity Building - Cooperative Agreement grant from the Centers for Disease Control and Prevention CDC) 5U50DP000721-04; and, NIH training grant T32 HL69764 (supporting CJB).

\author{
*Jawali Jaranilla \\ E-mail: jjaranil@jhsph.edu
}

